\title{
Domain Randomization for Macromolecule Structure Classification and Segmentation in Electron Cyro-tomograms
}

\author{
$1^{\text {st }}$ Chengqian Che* \\ Robotics Institute \\ Carnegie Mellon University \\ Pittsburgh, USA \\ cche@andrew.cmu.edu \\ $4^{\text {th }}$ Xin Gao \\ Computational Bioscience Research Center \\ King Abdullah University of Science and Technology \\ Thuwal, Saudi Arabia \\ xin.gao@kaust.edu.sa
}

\author{
$1^{\text {st }}$ Zhou Xian* \\ Robotics Institute \\ Carnegie Mellon University \\ Pittsburgh, USA \\ xianz1@andrew.cmu.edu
}

\author{
$3^{\text {rd }}$ Xiangrui Zeng \\ Computational Biology Department \\ Carnegie Mellon University \\ Pittsburgh, USA \\ xiangruz@andrew.cmu.edu
}

\author{
$5^{\text {th }}$ Min $\mathrm{Xu}^{+}$ \\ Computational Biology Department \\ Carnegie Mellon University \\ Pittsburgh, USA \\ mxu1@cs.cmu.edu
}

\begin{abstract}
It is crucial to study and understand cellular processes. In recent years, Cellular Electron CryoTomography (CECT) serves as a powerful 3D imaging tool to visualize spatial structure of macromolecules inside the cell. However, it is challenging to analyze the macromolecular structures in a systematic way due to nature of the structural complexity of subcellular components. Existing computational and deep learning based approaches suffer from limited scalability, discrimination ability and lack of accurate annotated CECT data. Training with cheap simulated data can alleviate this problem while facing new challenges of bridging the "reality gap" between synthetic training data and real testing data. In this paper, we tackle the tasks of macromolecule structure classification and segmentation in CECT images by adapting a simple but effective technique, domain randomization. We show that by combining deep neural models and domain randomization, we are able to achieve significant improvements of $35.21 \%$ and $46.34 \%$ in tasks of classification and semantic segmentation for real CECT data, comparing to the model trained only on syhthetic data that aims to faithfully reproduce real-world data distribution.
\end{abstract}

Index Terms-Cellular Electron Cryo-Tomography, Subtomogram Classification, Deep Learning, Domain Randomization

\section{INTRODUCTION}

Cells are basic units of living organisms. Macromolecules are nano-machines that play significant roles in cellular processes. In order to fully understand cellular processes, it is critical to know the structures and spatial organizations of macromolecules inside single cells. Traditionally, it has been

* Equal contribution.

+ Correspoinding author.

This work was supported in part by U.S. National Institutes of Health (NIH) grant P41 GM103712. XZ was supported by a fellowship from Carnegie Mellon University's Center for Machine Learning and Health. XG was supported by the King Abdullah University of Science and Technology (KAUST) Office of Sponsored Research (OSR) under Award No. BAS/1/1624, FCC/1/1976-18, FCC/1/1976-23, FCC/1/1976-25, and FCC/1/1976-26. very difficult to acquire such data. In recent years, Cellular Electron Cryo-Tomography (CECT) is becoming an emerging powerful 3D imaging tool to visualize spatial structures of macromolecules inside single cells with sub-molecular resolution while preserving their native states [1].

However, it remains challenging to classify and recover the macromolecular structures in a systematic way due to the nature of the structural complexity of subcellular components. Macromolecules interact with their neighbours dynamically and continuously in a crowded environment. Also, though imaging at sub-molecular resolution, low signal-to-noise ratio (SNR) and missing wedge effects are inherent to CECT imaging process, impairing the visualization quality. In addition, due to the process of structure determination using single-particle cryo-EM, current technical limitations require collecting very large datasets. BY taking the advantage of automatic image acquisition, electron microscope is able to capture hundreds of tomograms within several days. The image data contains millions of structurally highly heterogeneous macromolecules [2]. The tomograms can be further divided into cubic sub-volume called a subtomogram, which enclosing the extracted macromolucule. However, the existing computational approaches are severely limited in scalability and discrimination ability, which makes them incapable of automatically processing a large amount of data.

Previous works approached this problem with various techniques such as template matching [3], [4] and referencefree subtomogram classification averaging [1], [5], while they lack scalability due to heavy computation. Works with handdesigned features [6], on the other hand, cannot handle noise and missing wedge effect in the data. In recent years, deep learning based based approaches [7]-[9] have also been proposed and serves as a powerful tool in addressing problems 


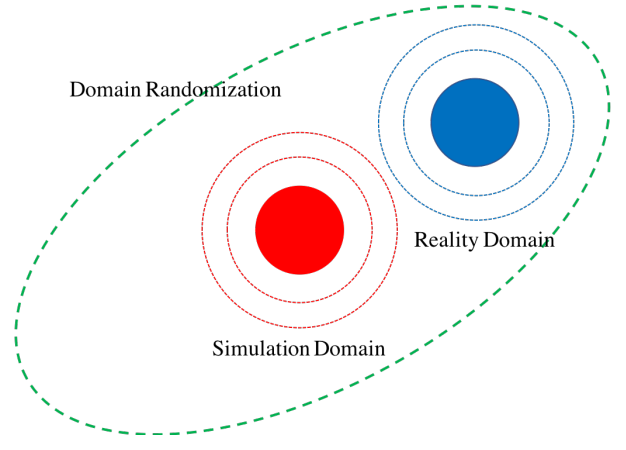

Fig. 1. Process of domain randomization: red and blue circles define the simulation dataset domain and reality dataset domain with a domain shift. Domain randomization (green dashed ellipse) bridges the gap between simulation and reality by providing high variability in simulation domain during training

in CECT images. In general, previous methods suffer from extremely expensive labor or computational cost for acquiring annotated CECT data. Training with cheap simulated data will greatly reduce this cost; however, bridging the "reality gap" between synthetic data and real-world data becomes a new challenge. Conventionally, deep neural models assume that distribution of training and testing data to be identical [10]. The differences between training (source domain) and testing (target domain) data distributions of input and output variables are defined as domain shift, which can severely bias a model's performance [11]. We will discuss related work in more details in Section II.

In this work, we adapt a recently proposed simple yet effective technique named domain randomization [12] to transfer models trained on simulated images to real images. Instead of training a model on a single simulated environment, we expose the model to an environment with high variability by randomizing the simulation process as shown in Figure 1. If the simulation has a significantly enough variability, the model trained with simulated data can show better generalizability to the real-world data with no additional training. We validate our method with both classification and semantic segmentation tasks for real CECT subtomogram data. Our results show that domain randomization boost up classification and segmentation results by $\mathbf{3 5 . 2 1 \%}$ and $\mathbf{4 6 . 3 4 \%}$ respectively. Our work demonstrates that it is now possible for model solely trained with cheap simulation data to be deployed in real-world tasks.

\section{RELATED WORK}

\section{A. Subtomogram Classification and Segmentation}

One popular line of previous work on localizing macromolecules uses template matching based methods [3], [4], while their capabilities are restricted to recovering known macromolecular structures, while it is desired to have model discovering new structures. Reference-free subtomogram averaging and alignment methods were also developed for recovering novel structures [1], [5], [13], [14], but they are not data-driven and require expensive computation process when operating in the $6 \mathrm{D}$ rigid transformation space. Also, their performance is hindered due to the fact that the crowded nature of tomograms makes it inevitable to include neighbouring complexes, which could bias similarity measures used in these methods. Rotation invariant feature [6] and pose normalization [15] were attempts to alleviate expensive computational cost, while they cannot handle well anisotropic resolution and noise present in data captures via CECT.

Deep learning based approaches [7]-[9] were also studied recently and showed promising results in both classification and semantic segmentation tasks. In 2017, Xu et al [7] first proposed a deep learning model DSRF3D for CECT image classification, demonstrating essential improvements in scalability and discrimination ability. Later, Che et al [8] extended the existing work to achieve higher classification accuracy by proposing 3 deep learning models. In 2018, Liu et al [9] developed a multi-task learning model for macromolecule segmentation, classification, and coarse structural recovery for CECT images. These models still suffer not only from high computational costs for obtaining large training dataset but also poor generalization in real-world data even if they are trained on simulated data.

\section{B. Bridging the Reality Gap}

To bridge the gap between simulation and the reality, studies in computer vision field have been focused on adapting deep learning models trained in a source domain to a new target domain [16]-[18]. There is a variety of methods utilizing domain adaption including re-training the model within the target domain [19] and adapting weights of the models in according to the statistics of the source and target domain. A mapping from the target domain to the source domain can be also learned [20]. Other related methods such as iterative learning control have also been applied to different robotic control problems including model car controling [21] and surgical robotics [22]. Our method adapts domain randomization, in contrast to those approaches, requiring no additional training on real-world data. In addition, by performing system identification and rendering high-quality photo-realistic images, there are approaches making the simulation closely match the physical reality. Unlike those methods, our method is very simple to understand and easy to implement. It uses low-quality simulation, making accessing a large amount of labeled training data can be done at a very low cost.

\section{METHODS}

Our goal is to bridge the reality gap by training deep neural networks using domain randomization. We explore the ability of domain randomization in both subtomogram classification and segmentation. In the remaining of this section, we discuss the process of generating simulated CECT data, how randomization is applied specifically and details about our network architecture.

\section{A. Synthetic Data Generation}

Similar to previous works, we generate the synthetic subtomograms by simulating the actual subtomogram reconstruction. The simulation takes into account for noise, missing 


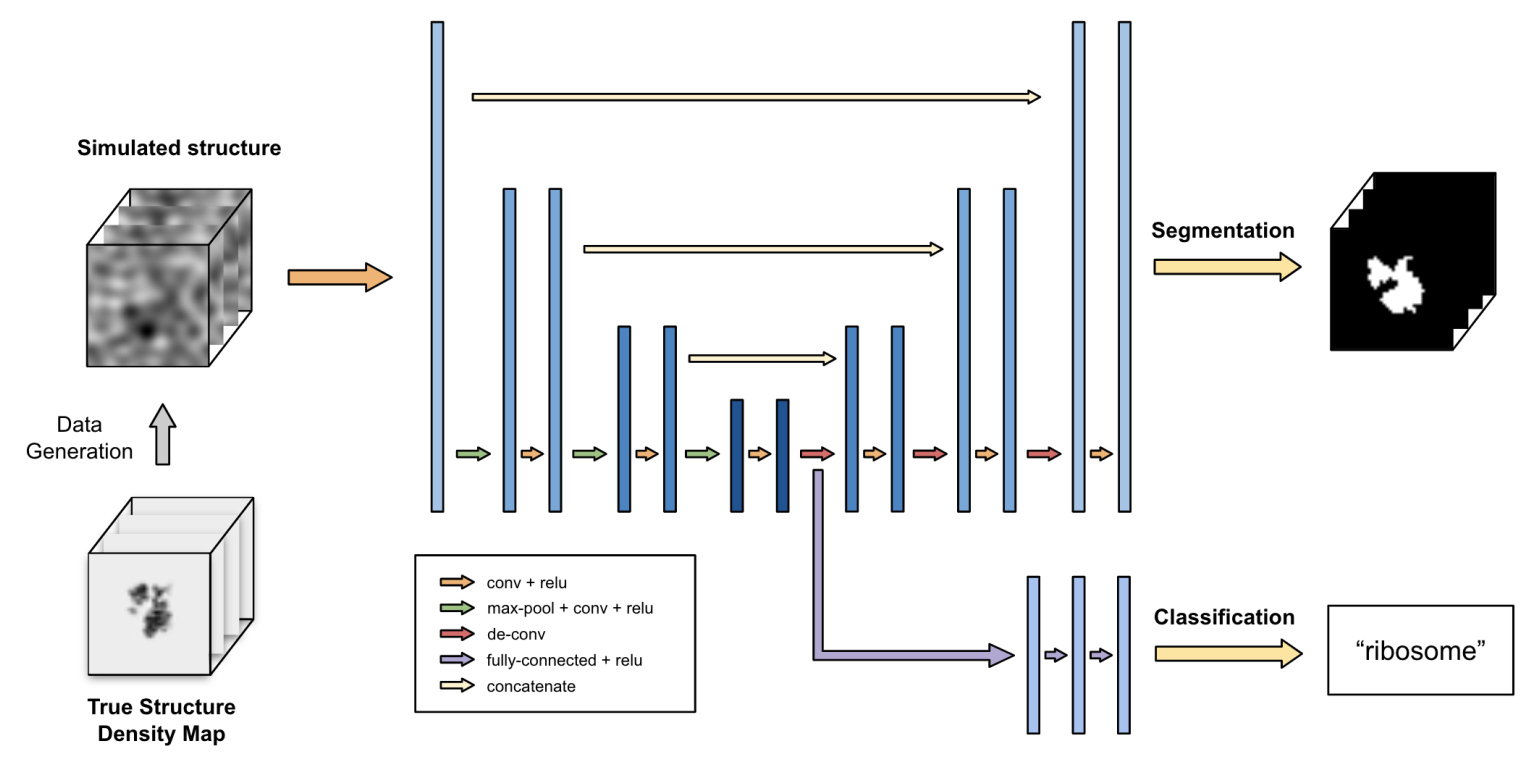

Fig. 2. Architecture Overview

wedge effect, as well as other electron optical factors. Two important factors are Contrast Transfer Function (CTF) and Modulation Transfer Function (MTF) which can be properly adjusted by the de-focus range (Dz) and spherical aberration(Cs). In the real world, noise is always the major limitation in CECT images. Thus, we need to include proper noise to our dataset to acquire desired SNRs, usually at a lower range. In additional to low SNR, CECT also has another major limitation, a limited range of observable tilt angles (also known as missing wedge angles) for common biological samples.

More specifically, the electron optical density of macromolecular complexes is proportional to the electrostatic potential. We generate the density maps using the PDB2VOL program from the Situs [23] package. These maps are in volumes of $40^{3}$ voxels with a resolution and voxel spacing of $1.368 \mathrm{~nm}$. Then we simulate electron subtomograms from density maps using a procedure derived from [24]. The simulation procedure is simplified in order to significantly speedup the simulation process which allows generating large amount of training data and directly feed into the CNN model on fly. To do so, we first include into the density map proper noises with desired SNR level and missing-wedge effects. Next we convolve the 3D images with the adjusted CTF and MTF to simulate optical effects in real data [25], [26]. The acquisition parameters are determined based on typical experimental tomograms [27]. The MTF is defined as $\operatorname{sinc}(\pi \omega / 2)$, in consistence with a realistic detector [28]. $\omega$ is the fraction of the Nyquist frequency. We simulate subtomograms of 4 types of macromolucules: ribosome, proteasome, TRiC and membrane. With the help of randomization, we can provide enough simulated variability for the network to generalize the real-world data at the test time.

\section{B. Domain Randomization in Data Generation}

As the key to the success of domain randomization, we need to provide the network with a highly variational simulated environment to train. We need to carefully design the simulated training environment so that it can enable effective transfer of the model to real-world settings. We use the simulation program discussed above, and randomize input variable in source domain. More specifically, we provide randomization in the following aspects with specific ranges:

- SNR: We uniformly sample the SNRs from 0.03 to 10 at a logarithmic scale.

- Missing wedge angle: We uniformly sample missing wedge angles from $0^{\circ}$ to $50^{\circ}$, corresponding to tile angle ranges from $\pm 90^{\circ}$ to $\pm 40^{\circ}$.

- Defocus (Dz): We uniformly sample from -12 to 0 .

- Spherical aberration (Cs): We uniformly sample from 1.5 to 3.0 .

- Augmentation: for each sample, we add additional augmentation to the simulated subtomograms based on its variance and mean.

The randomization of simulation parameters produces a very large diversity of subtomograms. Figure 3 shows examples of simulated subtomograms from different classes, generated by randomized inputs. The idea is that by forcing the model to handle a greater degree of variation in terms of wide range of SNRs and missing wedge angles etc, we can train a model that can generalize in real-world images, even though the simulated subtomograms are not entirely realistic. With the wider variation in parameters in simulation, it is more likely the model can learn to be robust to noises and capture more properties in the real world data. 


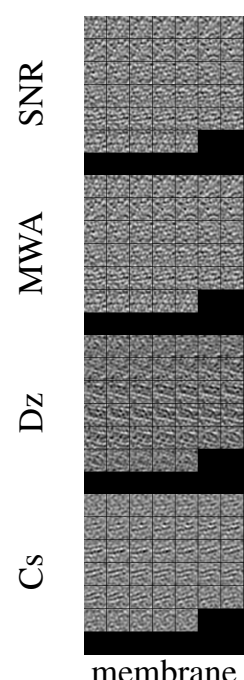

membrane

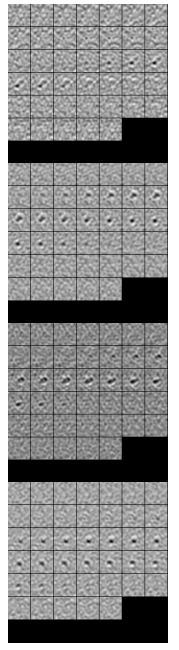

proteasome

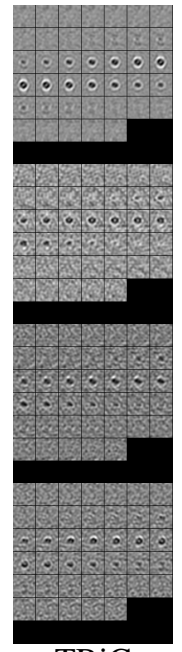

TRiC

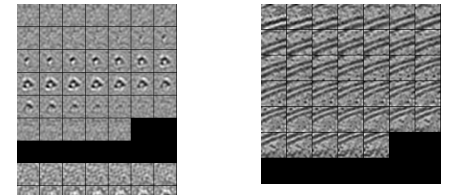

membrane

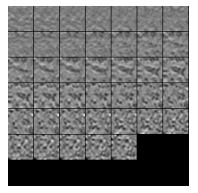

proteasome

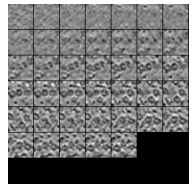

TRiC



ribosome

Fig. 4. Slices of examples of testing(real) subtomgorams of 4 structural classes

Note that for the purpose of this work, we did not spend too much effort trying to push the performance limit of the network architecture. Instead, we focus on evaluating how adopting domain randomization in data simulation can boost the performance. We choose such a simple network structure on purpose without any over-engineering tailored to our tasks, such that it is reasonable to expect any demonstrated performance boost is universally applicable to any network models. We leave investigation on optimal network architecture for our tasks as future work.

\section{EXPERIMENTS}

In this section, we discuss details about two experiments for both classification and segmentation tasks. In order to demonstrate the ability of domain randomization to bridge the reality gap between synthetic data and real-world data, we train the network proposed in section III-C with domain randomization in synthetic data generation. As for the baseline method, instead of randomly sampling input parameters (i.e. SNRs, missing wedge tilt angles, Dz and Cs), we manually set the parameters based on how we captured the real-world data. Missing wedge tilt angles, $\mathrm{Dz}$ and $\mathrm{Cs}$ are $30^{\circ},-6 \mu \mathrm{m}$ and $2.7 \mathrm{~mm}$ respectively. Baseline SNR is set to be 0.1 by measuring the testing data. The only "random" input for the baseline is the data augmentation. During training, we generate a batch of 32 subtomograms on the fly for each iteration. Samples for each batch are simulated with either randomization added, or based on fixed parameters for baseline. We standardize each batch with a global mean and standard deviation.

\section{A. Training on Synthetic Data}

For network training, we train both classification and segmentation branch simultaneously. In order to balance the loss, we add up the losses following: $\ell=\lambda \ell_{c l s}+(1-\lambda) \ell_{\text {seg }}$. Our results are reported with $\lambda=0.15$. We train our networks for 15000 iterations using stochastic gradient descent (SGD) optimizer with Nesterov momentum of 0.9 , and learning rate is set to be 5e-3. We implement our model in Pytorch [31] and train on a Linux workstation with two Nvidia GTX 1080 GPUs and 32 CPU cores.

\section{B. Testing on Real Dataset}

Since the goal is to demonstrate the generalization capability of model trained within simulated environment in real world, we use real CECT images for testing. The real dataset contains 1,051 subtomograms. The subtomograms are processed with template search [4] and then manually filtered from a rat neuron tomogram [32]. The tilt angle range was $-50^{\circ}$ to

\footnotetext{
${ }^{1}$ The figure provided is merely for illustration purpose. It does not reflect the exact number of layers in our model for the sake of best visualization.
} 


\begin{tabular}{|c|c|c|c|}
\hline Method & Classification Accuracy & Pixel Accuracy & mIoU \\
\hline \hline w/o domain randomization & 0.605 & 0.923 & 0.205 \\
w/ domain randomization & $\mathbf{0 . 8 1 8}$ & $\mathbf{0 . 9 4 0}$ & $\mathbf{0 . 3 0 0}$ \\
\hline
\end{tabular}

TABLE I

PERFORMANCE OF OUR MODEL WITH AND WITHOUT DOMAIN RANDOMIZATION

$+70^{\circ}$. The 1,051 subtomograms are of 4 classes: 80 ribosome subtomogram, 460 mitochondrial membrane subtomogram, 125 TRiC subtomogram, and 386 single capped proteasome subtomogram. Examples of real testing data are shown in Figure 4. At the test time, we smooth our testing data using a Gaussian filter data after a global standardization. We compare the performance between networks using domain randomization and the baseline method in Section V.

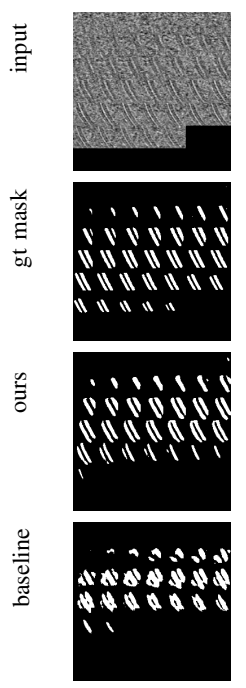

membrane
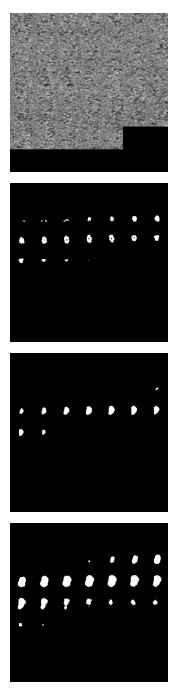

proteasome
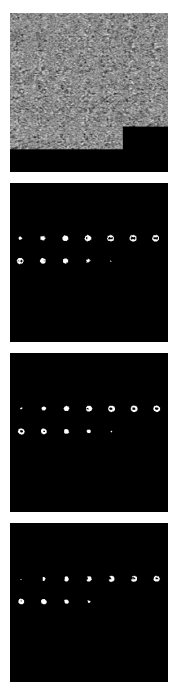

TRiC
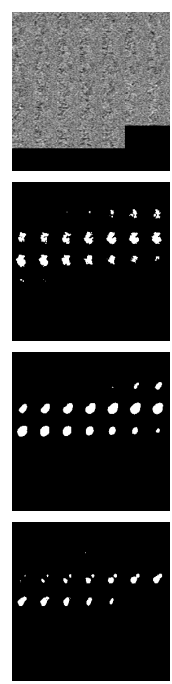

ribosome
Fig. 5. Visualizations of predicted segmentation masks. From top to bottom: input data, ground-truth mask, result of the model with domain randomization, and result of the baseline approach. The 3D images are plotted as slices.

\section{RESULTS}

In this section, we show results for both classification and segmentation tasks. We report accuracy for classification, and both pixel accuracy and mIoU for segmentation in Table I. We also present some visualized comparisons between the results produced by the model with domain randomization and the baseline, as shown in Figure 5. Note that for method with domain randomization, we report the mIoU by taking a standard probability threshold of 0.5 in the predicted segmentation mask, while for the baseline, we took a threshold of 0.2 , since the baseline model performs best with this value.

Our model trained with domain randomization reaches classification accuracy of 0.818 on real-world testing dataset, showing a huge improvement of $35.21 \%$ compared to the model without domain randomization. For segmentation, similarly, the model trained with domain randomization shows better performance in terms of pixel accuracy, and significantly outperforms the baseline model by $46.34 \%$ in terms of mIoU. Visual inspection reveals that with domain randomization, our model is more powerful at capturing fine-grained features and its predicted masks show clearer structures. Our results demonstrate that with the help of randomization in the source domain, the trained model is able to generalize in a highly diverse real-world scenarios for both classification and segmentation tasks. Due to the high variability provided to the model during training time, our model is able to cover most possible subtomograms scenes in real world.

\section{CONCLUSION}

Macromolecules are key components towards understanding cellular processes. In order to systematicly detect native structures and spatial organizations of macromolecules inside cells in situ, CECT has been developed to be the most promising technique in the past decades. However, CECT analysis is very difficult due to the large data quantity, high level of structural complexity, molecular crowding and imaging limitations in CECT data. High-throughput subtomogram classification and segmentation are key steps for significantly improving macromolecular structural recognition and recovery, by reducing of such structural complexity and crowding. Existing unsupervised geometry based subtomogram analysis methods often have poor scalability. Recently, deep learning based supervised subtomogram classification [7] and segmentation [9] potentially make a powerful technique for the large-scale subtomogram analysis with remarkable improvements in speed and accuracy. But large amount of structurally annotated subtomograms is generally necessary for the successful training of such methods. The preparation of such training data from the same tomogram dataset is generally laborious and computationally expensive. Therefore, training with virtually unlimited training data collected from simulation is a beneficial and practical solution. However, in such case, we will have to overcome the domain shift problem. This is because that the domain shift is likely to produce significantly biased the results in the cross data source prediction.

In this paper, we adapt a simple domain randomization framework for such cross-domain subtomogram structural classification and segmentation tasks. The simulation procedure is based on 3D convolution that is simplified from existing back-projection based simulation approaches. The randomization is implemented by varying the parameters that defines the models electron optical effects of transmission electron microscopy imaging. Our tests showed that our domain randomization method significantly improved crossdata source subtomogram classification and segmentation. This work demonstrates large potentials of fully utilizing the power of deep learning for large-scale macromolecule structural analysis. The optimal domain randomization and multi-branch 
CNN models and training strategies for better performance remain to be explored.

\section{REFERENCES}

[1] J. A. Briggs, "Structural biology in situthe potential of subtomogram averaging," Current opinion in structural biology, vol. 23, no. 2, pp. 261-267, 2013.

[2] C. M. Oikonomou and G. J. Jensen, "Cellular electron cryotomography: Toward structural biology in situ," Annual Review of Biochemistry, vol. 86, no. 1, pp. 873-896, 2017, pMID: 28426242. [Online]. Available: https://doi.org/10.1146/annurev-biochem-061516-044741

[3] M. Beck, J. A. Malmström, V. Lange, A. Schmidt, E. W. Deutsch, and R. Aebersold, "Visual proteomics of the human pathogen leptospira interrogans," Nature methods, vol. 6, no. 11, p. 817, 2009.

[4] S. Nickell, C. Kofler, A. P. Leis, and W. Baumeister, "A visual approach to proteomics," Nature reviews Molecular cell biology, vol. 7, no. 3, p. $225,2006$.

[5] A. Bartesaghi, P. Sprechmann, J. Liu, G. Randall, G. Sapiro, and S. Subramaniam, "Classification and $3 \mathrm{~d}$ averaging with missing wedge correction in biological electron tomography," Journal of structural biology, vol. 162, no. 3, pp. 436-450, 2008.

[6] M. Xu, W. Li, G. M. James, M. R. Mehan, and X. J. Zhou, "Automated multidimensional phenotypic profiling using large public microarray repositories," Proceedings of the National Academy of Sciences, vol. 106, no. 30, pp. 12323-12328, 2009.

[7] M. Xu, X. Chai, H. Muthakana, X. Liang, G. Yang, T. Zeev-BenMordehai, and E. P. Xing, "Deep learning-based subdivision approach for large scale macromolecules structure recovery from electron cryo tomograms," Bioinformatics, vol. 33, no. 14, pp. i13-i22, 2017

[8] C. Che, R. Lin, X. Zeng, K. Elmaaroufi, J. Galeotti, and M. Xu, "Improved deep learning-based macromolecules structure classification from electron cryo-tomograms," Machine Vision and Applications, vol. 29, no. 8, pp. 1227-1236, 2018.

[9] C. Liu, X. Zeng, R. Lin, X. Liang, Z. Freyberg, E. Xing, and M. Xu, "Deep learning based supervised semantic segmentation of electron cryo-subtomograms," in 2018 25th IEEE International Conference on Image Processing (ICIP). IEEE, 2018, pp. 1578-1582.

[10] T. Tommasi, M. Lanzi, P. Russo, and B. Caputo, "Learning the roots of visual domain shift," CoRR, vol. abs/1607.06144, 2016. [Online]. Available: http://arxiv.org/abs/1607.06144

[11] J. Quionero-Candela, M. Sugiyama, A. Schwaighofer, and N. D. Lawrence, Dataset Shift in Machine Learning. The MIT Press, 2009.

[12] J. Tobin, R. Fong, A. Ray, J. Schneider, W. Zaremba, and P. Abbeel, "Domain randomization for transferring deep neural networks from simulation to the real world," in 2017 IEEE/RSJ International Conference on Intelligent Robots and Systems (IROS). IEEE, 2017, pp. 23-30.

[13] S. H. Scheres, R. Melero, M. Valle, and J.-M. Carazo, "Averaging of electron subtomograms and random conical tilt reconstructions through likelihood optimization," Structure, vol. 17, no. 12, pp. 1563-1572, 2009.

[14] M. Xu, M. Beck, and F. Alber, "High-throughput subtomogram alignment and classification by fourier space constrained fast volumetric matching," Journal of structural biology, vol. 178, no. 2, pp. 152-164, 2012.

[15] X.-P. Xu, C. Page, and N. Volkmann, "Efficient extraction of macromolecular complexes from electron tomograms based on reduced representation templates," in International Conference on Computer Analysis of Images and Patterns. Springer, 2015, pp. 423-431.

[16] B. Kulis, K. Saenko, and T. Darrell, "What you saw is not what you get: Domain adaptation using asymmetric kernel transforms." in CVPR. IEEE Computer Society, 2011, pp. 1785-1792. [Online]. Available: http://dblp.uni-trier.de/db/conf/cvpr/cvpr2011.htmlKulisSD11

[17] J. Hoffman, E. Rodner, J. Donahue, K. Saenko, and T. Darrell, "Efficient learning of domain-invariant image representations," in $I C L R$, Y. Bengio and Y. LeCun, Eds., 2013. [Online]. Available: http://dblp.uni-trier.de/db/conf/iclr/iclr2013.htmlabs-1301-3224

[18] J. Hoffman, S. Guadarrama, E. S. Tzeng, R. Hu, J. Donahue, R. Girshick, T. Darrell, and K. Saenko, "Lsda: Large scale detection through adaptation," in Advances in Neural Information Processing Systems 27, Z. Ghahramani, M. Welling, C. Cortes, N. D. Lawrence, and K. Q. Weinberger, Eds. Curran Associates, Inc., 2014, pp. 3536-3544. [Online]. Available: http://papers.nips.cc/paper/5418-1sdalarge-scale-detection-through-adaptation.pdf
[19] J. Yosinski, J. Clune, Y. Bengio, and H. Lipson, "How transferable are features in deep neural networks?" in NIPS, Z. Ghahramani, M. Welling, C. Cortes, N. D. Lawrence, and K. Q. Weinberger, Eds., 2014, pp. 3320-3328. [Online]. Available: http://dblp.unitrier.de/db/conf/nips/nips2014.htmlYosinskiCBL14

[20] Y. Li, N. Wang, J. Shi, J. Liu, and X. Hou, "Revisiting batch normalization for practical domain adaptation." CoRR, vol. abs/1603.04779, 2016. [Online]. Available: http://dblp.unitrier.de/db/journals/corr/corr1603.htmlLiWSLH16

[21] P. Abbeel, M. Quigley, and A. Y. Ng, "Using inaccurate models in reinforcement learning," in International Conference on Machine Learning (ICML) Pittsburgh PA, USA, 2006.

[22] J. van den Berg, S. Miller, D. Duckworth, H. Hu, A. Wan, X.-Y. Fu, K. Y Goldberg, and P. Abbeel, "Superhuman performance of surgical tasks by robots using iterative learning from human-guided demonstrations." in ICRA. IEEE, 2010, pp. 2074-2081. [Online]. Available: http://dblp.unitrier.de/db/conf/icra/icra2010.htmlBergMDHWFGA10

[23] W. Wriggers, R. Milligan, and J. McCammon, "Situs: A Package for Docking Crystal Structures into Low-Resolution Maps from Electron Microscopy," Journal of Structural Biology, vol. 125, no. 2-3, pp. 185$195,1999$.

[24] F. Förster, S. Pruggnaller, A. Seybert, and A. Frangakis, "Classification of cryo-electron sub-tomograms using constrained correlation," Journal of structural biology, vol. 161, no. 3, pp. 276-286, 2008.

[25] J. Frank, Three-dimensional electron microscopy of macromolecular assemblies. Oxford University Press, New York, 2006.

[26] S. Nickell, F. Förster, A. Linaroudis, W. Net, F. Beck, R. Hegerl, W. Baumeister, and J. Plitzko, "TOM software toolbox: acquisition and analysis for electron tomography," Journal of Structural Biology, vol. 149, no. 3, pp. 227-234, 2005.

[27] T. Zeev-Ben-Mordehai, D. Vasishtan, A. H. Durán, B. Vollmer, P. White, A. P. Pandurangan, C. A. Siebert, M. Topf, and K. Grünewald, "Two distinct trimeric conformations of natively membrane-anchored fulllength herpes simplex virus 1 glycoprotein b," Proceedings of the National Academy of Sciences, vol. 113, no. 15, pp. 4176-4181, 2016.

[28] G. McMullan, S. Chen, R. Henderson, and A. Faruqi, "Detective quantum efficiency of electron area detectors in electron microscopy," Ultramicroscopy, vol. 109, no. 9, pp. 1126-1143, 2009.

[29] O. Ronneberger, P. Fischer, and T. Brox, "U-net: Convolutional networks for biomedical image segmentation," in International Conference on Medical image computing and computer-assisted intervention. Springer, 2015, pp. 234-241.

[30] K. He, G. Gkioxari, P. Dollár, and R. Girshick, "Mask r-cnn," in Proceedings of the IEEE international conference on computer vision, 2017, pp. 2961-2969.

[31] A. Paszke, S. Gross, S. Chintala, G. Chanan, E. Yang, Z. DeVito, Z. Lin, A. Desmaison, L. Antiga, and A. Lerer, "Automatic differentiation in pytorch," in NIPS-W, 2017.

[32] Q. Guo, C. Lehmer, A. Martínez-Sánchez, T. Rudack, F. Beck, H. Hartmann, M. Pérez-Berlanga, F. Frottin, M. S. Hipp, F. U. Hartl et al., "In situ structure of neuronal c9orf72 poly-ga aggregates reveals proteasome recruitment," Cell, vol. 172, no. 4, pp. 696-705, 2018. 